Jerzy E. Kiwerski

\title{
The Application of Biomechanics on Orthopaedic Rehabilitation
}

Department of Warsaw College of Rehabilitation, Poland

Corresponding Author : Jerzy E. Kiwerski, Department of Warsaw College of Rehabilitation, Poland. E-mail: jerzy.kiwerski@wsr.edu.pl

Received date: July 24,2019; Accepted date : July 29,2019; Published date: July 31,2019.

Citation: Jerzy E. Kiwerski, (2019) The Application of Biomechanics on Orthopaedic Rehabilitation. J. Orthopaedics and Surgical Sports Medicine,2(1) : Doi:10.31579/2641-0427/015

Copyright : (c) 2019 Jerzy E. Kiwerski. This is an open-access article distributed under the terms of The Creative Commons Attribution License, which permits unrestricted use, distribution, and reproduction in any medium, provided the original author and source are credited.

Development in medicine in large scale merits the development of biomechanical and biomedical engineering. This highly concerns with the medical rehabilitation, which according to the modern understanding is the interdisciplinary management aiming at recovering or improving efficiency of the organism which is beyond the ability of pure medical [1]. Model of Polish rehabilitation is based on four basic principles: early application of rehabilitation, it's universality, complexity and continuity procedures. Early application of rehabilitation prevents from development of unwanted changes such as: contractures, muscular atrophies, trophic abnormalities and decreases the frequent negative outcomes due to long term immobilization [2].Complexion principles defines then interdisciplinary character, paying attention at the importance increasing maximum care to improve the activities disabled, supplying him with necessary prosthetic - orthotic equipment in order to easy the performance of activities at home conditions, environment and if possible - come back to professional job. Continuity rehabilitation concerns generally to people with marked, long-lasting dysfunctions of the body, who require different forms of rehabilitation often for several months or years. Continuity is also very important in application of orthotic equipment. It mainly concerns with cripple children, who are equipped in prosthesis, orthopedic footwear's, orthotics and who require permanent modifications in the course development, growth [3].Biomechanical researches started in Poland in thirties last century concerned mainly on biomechanics of sports and in little consideration - orthopedic. At present it's difficult imagine the function and further development of several disciplines without close collaboration and application of the biomechanical discoveries in wide range application starting from issues concerned with explaining pathomechanics damages spine, hip joints, knees, diagnosis, by practical application of the rehabilitation, prosthetic equipment's, orthotics, application of functional electrostimulation to the composition of the objective methods to evaluate applied procedures, the improve to stereotype posture, gait, locomotion [4].In my paper I would like to refer one narrow topic concerning with the issue of functional electrostimulation in improving the lost functions following the damage of the central nervous system. At present there is a wide application of the apparatus to stimulate the function of the lower extremity of the patients with hemiplegia [5]. It is made up of Surface electrode stimulator positioned over the peroneal nerve and connected to the channel with a stimulator. During the gate at the moment unloading of the patients lower limb they open local contact in heel, which allows the sending of the impulses to the stimulating electrode in which the irritated peroneal nerve causes the contraction of the fibular muscles, and as a result - dorsal flexion and elevation of the outer edge of the foot. This allows the smooth raising without hooking the ground.In the seventies of last century in the Rehabilitation Centre in Konstancin with the cooperation of the team of Warsaw Polytechnic University [6], an experiment was done to apply functional electrostimulation in the rehabilitation of patients with tetraplegią (figure).
In such cases (the hand is paralyzed) - median nerve is stimulated (steering function of the fingers flexion and opposition of the thumb) including stimulation of the radial nerve (supplying extensors of the wrist and fingers) in order to enforce the function of the paralyzed hand. This led to the construction of the prototype hybrid orthosis to enable the simple functions of grasping of the paralyzed hand. For the stimulation, there was an application of a stimulators implanted under the skin of the forearm in which electrodes were put on the median and radial nerve. Required program of the motion during the time of performing activ grasping activities is controlled by the steering device [7].Partially mentioned selected problems and the application of functional electrostimulation show the importance and extent of topics dealt by biomechanics, indicating its close relationship with medicine, showing how more are still to be done in this field study.

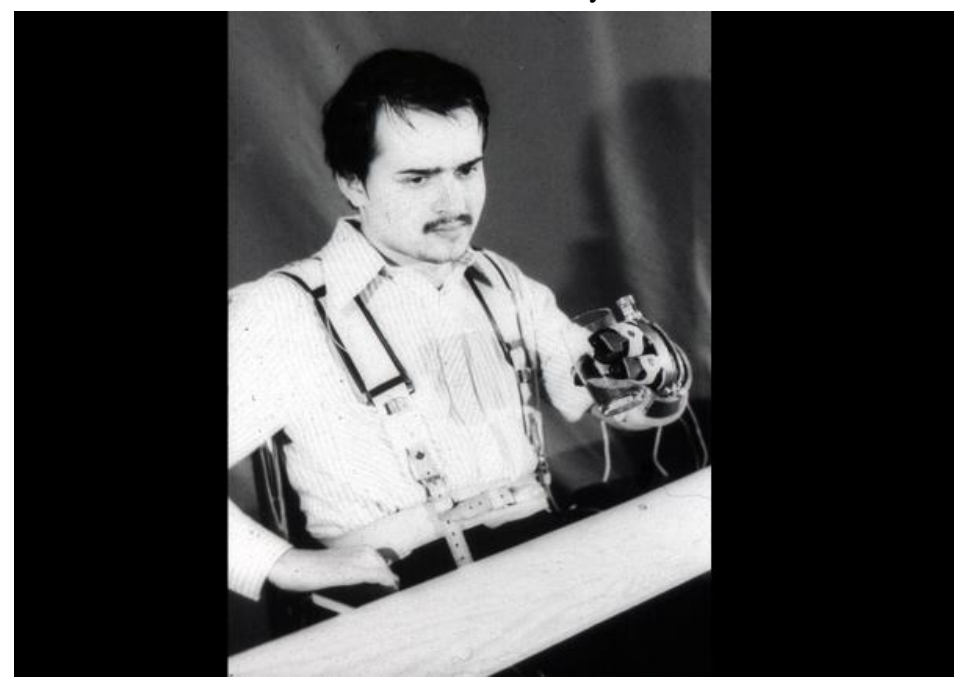

Figure 1: Hybrid orthosis to enable the simple function of grasping of the paralyzed hand.

\section{References}

1. Kiwerski J.E. (2003) Medical Rehabilitation. Ed. Medical University Warsaw.

2. Kiwerski J.E. 2005 Medical Rehabilitation. PZWL, Warszawa.

3. Kiwerski J.E. (2014) Spinal disorders and surgeries. PZWL, Warszawa.

4. Kiwerski J.E. (2012) General Physiotherapy. PZWL Warszawa.

5. Kiwerski J.E. (2016) Treatment of craniocerebral injuries. In: Opara W, Craniocerebral injuries. PZWL, Warszawa,

6. Paśniczek $\mathrm{R}$, function of the human extremities by functional electrical stimulation. Pol J Phys Eng. Kiwerski J.E. (2004) Supporting of the lost 10:43 -53.

7. Kiwerski J.E, Paśniczek R. Będziński R, Kędzior K, Kiwerski J.E. et al. (2004) Biomechanics and Rehabilitation Engineering. Ed. Exit, Warszawa, 581- 612 . 